\title{
Preserving hepatic artery flow during portal triad blood occlusion improves regeneration of the remnant liver in rats with obstructive jaundice following partial hepatectomy
}

\author{
ZHE KONG ${ }^{1}$, JIAN-JUN HU ${ }^{2}$, XIN-LAN GE ${ }^{1}$, KE PAN $^{1}$, CHONG-HUI LI $^{1}$ and JIA-HONG DONG ${ }^{3}$ \\ ${ }^{1}$ Institute of Hepatobiliary Surgery, Chinese PLA General Hospital, Chinese PLA Medical School, Beijing 100853; \\ ${ }^{2}$ Former Outpatient Department for Bureau of Information Communication, Agency for Offices Administration, \\ Central Military Commission, Beijing 100840; ${ }^{3}$ Center for Hepatopancreatobiliary Diseases, \\ Beijing Tsinghua Changgung Hospital, Tsinghua University, Beijing 102218, P.R. China
}

Received November 30, 2017; Accepted May 25, 2018

DOI: $10.3892 /$ etm.2018.6402

\begin{abstract}
In certain cases, major hepatectomy is essential and inevitable in patients with hilar cholangiocarcinoma and obstructive jaundice (OJ). The current study was designed to evaluate effects of a novel method of portal blood occlusion, where the portal vein was occluded (OPV) and the hepatic artery flow was preserved in rats with OJ that underwent partial hepatectomy. OJ was induced in rats by ligation of the common bile duct for 7 days. Subsequently, OJ rats underwent hepatectomy removing $76 \%$ of the liver following occlusion of the portal triad (OPT), OPV or without portal blood occlusion. Liver blood flow (LBF), liver damage and regeneration were assessed. The safety limit for the duration of liver ischemia was $20 \mathrm{~min}$ for OPT and $40 \mathrm{~min}$ for OPV in rats with OJ. OPT and OPV methods resulted in significantly decreased microvascular LBF in rats with OJ from 529.53 \pm 91.55 laser speckle perfusion units (LSPU) in the control to $136.89 \pm 32.32$ and $183.99 \pm 49.25 \mathrm{LSPU}$, respectively. Liver damage was assessed analyzing levels of serum alanine transaminase and direct bilirubin, determining interleukin-1 $\beta$ and tumor necrosis factor- $\alpha$ expression and histological examination. It was demonstrated that liver damage and caspase-3 and -9 expression in the liver were substantially reduced in the OPV group compared with the OPT group. In addition, the OPV method significantly improved liver regeneration in OJ rats, as
\end{abstract}

Correspondence to: Dr Chong-Hui Li, Institute of Hepatobiliary Surgery, Chinese PLA General Hospital, Chinese PLA Medical School, 28 Fuxing Road, Beijing 100853, P.R. China

E-mail: lich_plagh@163.com

Professor Jia-Hong Dong, Center for Hepatopancreatobiliary Diseases, Beijing Tsinghua Changgung Hospital, Tsinghua University, 168 Litang Road, Beijing 102218, P.R. China

E-mail: dongjiahong@mail.tsinghua.edu.cn

Key words: obstructive jaundice, hepatectomy, portal occlusion, laser speckle contrast imaging, hepatic artery indicated by increased rates of liver regeneration and expression of proliferating cell nuclear antigen and Ki-67 compared with the OPT group. Therefore, the OPV method may prolong the duration of portal blood occlusion, reduce liver injury and improve liver regeneration by preserving hepatic arterial flow during portal blood control in rats with OJ undergoing partial hepatectomy. The current study describes a novel technique, which may be applied in liver surgery in patients with complex jaundice.

\section{Introduction}

Obstructive jaundice (OJ) is a common clinical symptom in cholangiocarcinoma and pancreatic cancer, which usually impairs liver function and reduces the regenerative capacity of liver (1). Pathological changes resulting from OJ, including cholangitis, cholestatic liver injury and digestive disorders, may exhibit adverse effects on surgical outcomes (2). However, major hepatectomy is essential and inevitable in a number of patients, particularly in those with hilar cholangiocarcinoma and OJ. The degree of ischemia-reperfusion (I/R) injury and the function of the remnant liver have been key focuses during hepatectomy and are associated with post-operative morbidity and mortality (3). I/R injury induces formation of reactive oxygen species that cause oxidative stress and cell death, ultimately leading to a sterile inflammatory response that causes hepatocellular damage and liver dysfunction that can result in acute liver failure in most severe cases (4). The current treatment strategy is to perform biliary drainage prior to surgery and to minimize the scale of the surgical procedure (1). However, the outcome is not favorable. Systematic review and meta-analysis have revealed that preoperative biliary drainage was associated with increased postoperative morbidity and wound infection (5). As previously established, using a suitable method for the occlusion of hepatic blood inflow has a marked effect in reducing blood loss and alleviating liver damage following liver resection (4). The current study hypothesized that the method of occlusion of hepatic blood inflow affects the outcomes of hepatectomy in patients with OJ. 
The Pringle maneuver, occlusion of the portal triad (OPT), has been acknowledged for its ability in reducing blood loss during liver resection, and severe I/R injury is inevitable if the operation is complicated and prolonged occlusion is required $(6,7)$. In addition, it has been demonstrated that the icteric liver exhibits reduced tolerance for I/R injury $(8,9)$. However, the majority of cholangiocarcinoma cases coexist with OJ (10). At present, numerous occlusion strategies, including selective hemihepatic vascular occlusion, step-by-step vascular control and ischemic preconditioning have been designed to control the liver portal blood and reduce I/R injury (11-13). A method for occlusion of the portal vein (OPV), while preserving hepatic artery blood flow during liver surgery was initially proposed and was demonstrated to be effective in a rat model of normothermic liver I/R (14). Although partial hepatectomy is a good model for liver regeneration, the liver injury induced by predisposed OJ and operation-related I/R may lead to suppressed liver regeneration (7-9). Proliferating cell nuclear antigen (PCNA) and Ki-67 labeling indexes are two nuclear antigens associated with hepatocyte proliferation, which are often used to characterize the hepatic regenerative response (15). The current study was designed to evaluate the protective degree of OPV on the liver regeneration of OJ rats following partial hepatectomy.

\section{Materials and methods}

Animals and experimental model of OJ. A total of 216 male Sprague-Dawley rats (age, 6-8 weeks; weight, 200-220 g), were obtained from the Experimental Animal Center of the Academy of Military Medical Science (Beijing, China). Animals were fed standard chow with water ad libitum and kept at $24^{\circ} \mathrm{C}$ and $40 \%$ humidity with a $12 \mathrm{~h}$ light/dark cycle. OJ was induced by ligation of the common bile duct (CBD). To ensure the accuracy of the procedure, an operating microscope for small animal surgery was used (GX.SS.22-3, Binocular Operation Microscope; Shanghai Medical Optical Instruments Factory Co., Ltd, Shanghai, China). The current study was conducted in accordance with the guidelines set by the Guide for the Care and Use of Laboratory Animals (16) and was approved by the Ethical and Research Committee of the Chinese PLA Medical School (Beijing, China).

Prior to surgery, rats were fasted overnight with free access to water. Anesthesia was performed by isoflurane/oxygen inhalation (4\% isoflurane for induction, $1.5 \%$ for maintenance of anesthesia). Hair on the abdomen of the rats was removed and an upper midline abdominal incision of $\sim 3 \mathrm{~cm}$ was made following sterilization. The $\mathrm{CBD}$ was exposed and two 8-0 monofilament, non-absorbable nylon sutures (Shanghai Pudong Jinhuan Medical Products Co., Ltd, Shanghai, China) were placed under the bile duct between branches and anastomoses of CBD and pancreas. The sutures were ligated and the CBD between the two sutures was dissected. The abdominal wall was closed with a continuous suture. A second surgery was performed on rats with OJ following 7 days (17).

Experimental groups. Rats with OJ were randomly assigned into three groups: C group, sham operation/control group; OPT group, rats with OPT, including portal vein and hepatic artery; and OPV group, rats with OPV and preservation of the hepatic artery. The duration of occlusion was set to $15,20,30$, 40, 50 and $60 \mathrm{~min}$, and the 7-day survival rate following the second surgery was investigated in the OPT and OPV group $(n=10$ for each time point in each group, total $n=120)$. The duration of occlusion was set to $30 \mathrm{~min}$ for liver injury and liver regeneration studies, and the sample time points were at $6,24,72 \mathrm{~h}$ and 7 days post hepatectomy ( $\mathrm{n}=24$ for $\mathrm{C}$ group, $\mathrm{n}=24$ for OPV group and $\mathrm{n}=36$ for OPT group). A total of 12 rats were used for the measurement of liver blood flow.

Surgical procedures for partial liver ischemia and hepatectomy in rats with OJ. After a total of 7 days following the surgery inducing $O J$ in rats, a second surgery was performed. The procedure combined biliary recanalization and partial liver ischemia under portal blood bypass through the caudate lobe (14) and a partial hepatectomy was performed in all rats. Rats with OJ were anesthetized as described above. The abdomen was entered through the incision of the previous procedure and dilated CBDs were isolated. A cannula $(8 \times 0.8 \mathrm{~mm})$ was inserted into the duodenal side of the original bile duct and fixed. The second tip of the cannula was inserted into the dilated CBD and fixed.

When the recanalization of the bile duct was completed, the left and median liver lobes, which were resected in following steps, were ligated with 3-0 silk sutures. The pedicle, for the OPT group, or the portal vein, for the OPV group, of the right liver lobe was dissected and clamped with a microvascular clamp. In the $\mathrm{C}$ group, the pedicle was dissected but not clamped. The clamping was maintained for 15-60 min. During ischemia, the abdomen was covered with plastic film to prevent evaporation of body fluids. Following occlusion, clamps were removed and liver reperfusion was initiated. The left, median and caudate lobes were resected and the abdomen was closed with 3-0 sutures. Following surgery, all rats were provided with rodent chow and water ad libitum until 6, 24, $72 \mathrm{~h}$ and 7 days post hepatectomy.

Sample collection. Serum and liver tissue samples were collected at $6,24,72 \mathrm{~h}$ and 7 days following partial hepatectomy ( $n=6$ for each time point of each group). Serum samples from rats with $\mathrm{OJ}$ that did not undergo the second surgery were also collected for comparison $(n=6)$. All rats were anesthetized as mentioned above and $5 \mathrm{ml}$ blood was obtained from the inferior vena cava for serum testing. Then $\geq 2 \mathrm{ml}$ blood was drawn to sacrifice the rats weighing $200-220 \mathrm{~g}$ by exsanguination. The liver were resected and weighted. The blood samples were centrifuged at $2,500 \mathrm{x} \mathrm{g}$ at $4^{\circ} \mathrm{C}$ for $15 \mathrm{~min}$. The supernatant was stored at $-80^{\circ} \mathrm{C}$ for serum tests. Tissue samples that were taken from the remnant liver were cut into two pieces. One piece was immediately frozen in liquid nitrogen and stored at $-80^{\circ} \mathrm{C}$ for detection of gene and protein expression, the other piece was further sliced and fixed at $4^{\circ} \mathrm{C}$ for $24 \mathrm{~h}$ with $10 \%$ formaldehyde in 0.1 M phosphate buffer ( $\mathrm{pH} 7.4$ ) for histopathological study.

Measurement of blood flow of the hepatic artery and portal vein. A total of 6 normal and 6 rats with OJ were paired according to body weight. Blood flow of the hepatic artery (HAF) and portal vein (PVF) was measured using the Transonic T206 Flow Meter (Transonic Systems Inc., Ithaca, NY, USA) during the second surgery. Following anesthesia 
and laparotomy, the common portal pedicle of the liver was dissected and a suitable probe was placed around the hepatic artery and portal vein. Blood flow was monitored for $1 \mathrm{~min}$.

Measurement of microvascular liver blood flow (LBF) using laser speckle contrast imaging. Microvascular LBF in rats with OJ was assessed using laser speckle contrast imaging (moorFLPI-2 Full-Field Laser Perfusion Imager; Moor Instruments, Axminster, UK) during the second operation. Following PVF and HAF measurements, LBF of the whole liver was measured as previously described (18). Following ligation of the left and median liver lobes and clamping of the pedicle for the OPT group, or the portal vein for the OPV group, of the right liver lobe, the LBF of the right liver lobe was measured $5 \mathrm{~min}$ post-clamping. The sampling frequency was $21 \mathrm{images} / \mathrm{sec}$ and the image acquisition rate was 1 frame/sec in the normal resolution mode. The duration of recording was 15 sec. MoorFLPI-2 Review (version 4.0; Moor Instruments) was used to analyze the images and quantify the perfusion in arbitrary laser speckle perfusion units (LSPU).

Liver function test. Serum levels of alanine transaminase (ALT) and direct bilirubin (DBIL) were used as general markers of liver injury, which was measured with a serum analyzer (Cobas-Mira Plus; Roche Diagnostics $\mathrm{GmbH}$, Mannheim, Germany).

Reverse transcription-quantitative polymerase chain reaction $(R T-q P C R)$. Total RNA was extracted from frozen liver tissues using the RNA Simple Total RNA kit (Tiangen Biotech Co., Ltd., Beijing, China) according to the manufacturer's protocol. RNA (4 $\mu \mathrm{g})$ was reverse transcribed using the RevertAid First Strand cDNA Synthesis kit and Oligo-dT primers (Thermo Fisher Scientific, Inc., Waltham, MA, USA). qPCR was performed with the SYBR Premix Ex Taq II (Takara Bio, Inc., Otsu, Japan) and the StepOnePlus Real-Time PCR system (Applied Biosystems; Thermo Fisher Scientific, Inc.). Primers used were as follows: Tumor necrosis factor (TNF)- $\alpha$ forward, 5'-AAATGGGCTCCCTCTCATCAGTTC-3' and reverse, 5'-TCTGCTTGGTGGTTTGCTACGAC-3'; interleukin (IL)-1 $\beta$ forward, 5'-CACCTCTCAAGCAGAGCA CAG-3' and reverse, 5'-GGGTTCCATGGTGAAGTCAAC-3'; hypoxanthine phosphoribosyltransferase (HPRT) forward, 5'-GCTGAAGATTTGGAAAAGGTG-3' and reverse, 5'-AAT CCAGCAGGTCAGCAAAG-3'. The relative gene expression was normalized to HPRT. Values were calculated using the $2^{-\Delta \Delta \mathrm{Cq}}$ method as previously described and expressed as fold change vs. normal rat (19).

Histological assessment and Ki-67 immunohistochemistry. Formalin-fixed tissues were embedded in paraffin and $5-\mu \mathrm{m}$ sections were cut. Sections were stained for $3 \mathrm{~min}$ with hematoxylin and $2 \mathrm{~min}$ with eosin at room temperature for histological examination using a light microscope (magnification, x400). For immunohistochemistry, sections were incubated with a mouse anti-Ki-67 monoclonal antibody (1:50; cat. no. 550609; BD Biosciences, Franklin Lakes, NJ, USA) according to the manufacturer's protocol. Samples were counterstained with hematoxylin for $2 \mathrm{~min}$ at room temperature. The percentage of Ki-67-positive hepatocytes was determined in ten random visual fields using a light microscope (Leica Microsystems GmbH, Wetzlar, Germany) at magnification, x400 and the Ki-67 labeling index. All histological analyses were performed in a blinded manner with respect to the experimental groups.

Evaluation of liver regeneration following hepatectomy. Liver regeneration was evaluated using the rate of regeneration following hepatectomy of rats with OJ. Rate of regeneration=actual remnant liver weight at test point/W $\mathrm{x} 100 \%$, where $\mathrm{W}$ represents the weight of the whole liver prior to hepatectomy. W was calculated as weight of the resected liver $76 \%$ (the weight of the resected liver lobes account for $76 \%$ of the whole liver) (20).

Western blot analysis. Frozen liver samples were homogenized in radioimmunoprecipitation assay buffer (Cell Signaling Technology, Inc., Danvers, MA, USA) supplemented with $1 \mathrm{mM}$ phenyl methane sulfonyl fluoride and $1 \mathrm{X}$ phosphatase inhibitor. Homogenates were centrifuged at 10,000 x g for $10 \mathrm{~min}$ at $4^{\circ} \mathrm{C}$. Supernatants were collected and protein concentrations were determined using the bicinchoninic acid method. Samples containing $50 \mu \mathrm{g}$ of protein were separated on $10 \%$ SDS-PAGE gels and transferred to polyvinylidene difluoride membranes. Membranes were blocked with 5\% skimmed milk for $1 \mathrm{~h}$ at room temperature and incubated overnight at $4^{\circ} \mathrm{C}$ with primary antibodies: A mouse monoclonal antibody of anti-proliferating cell nuclear antigen (PCNA; 1:200; cat. no. sc-56; Santa Cruz Biotechnology, Inc., Dallas, TX, USA), rabbit anti-caspase-3 (Asp175) (5A1E) monoclonal antibody (cat. no. 9664) and rabbit anti-caspase-9 (Asp353) polyclonal antibody (cat. no. 9507) (both 1:1,000; Cell Signaling Technology, Inc.). Following, membranes were incubated with horseradish peroxidase-conjugated goat anti-mouse (sc-2005) or goat anti-rabbit (sc-2004) secondary antibodies $(1: 2,000$; Santa Cruz Biotechnology, Inc.) for $1 \mathrm{~h}$ at room temperature. Blots were developed with Super-Signal chemiluminescent substrate (cat. no. 34580; Thermo Fisher Scientific, Inc.). Blots were analyzed against mouse anti-GAPDH monoclonal antibodies (1:2,000; cat. no. KM9002T; Sungene Biotech Co., Ltd., Tianjin, China) expression, which was used as loading control.

Statistical analysis. Data are expressed as the mean \pm standard deviation. Student's t-test was used for the comparison of two groups and one-way ANOVA with Student-Newman-Keuls test was used for the comparison of three groups. SPSS (version 17.0; SPSS, Inc., Chicago, IL, USA) was used for analyses. $\mathrm{P}<0.05$ was considered to indicate a statistically significant difference.

\section{Results}

Preservation of hepatic artery flow during liver blood inflow control extends the duration of liver ischemia in rats with OJ. All rats with OJ survived until the second surgical procedure. The dilated CBDs with a diameter of 8-10 $\mathrm{mm}$ were visible during the second laparotomy. To assess the tolerance of rats with $\mathrm{OJ}$ to liver ischemia and hepatectomy, the safe limit for the duration of liver ischemia in rats with $\mathrm{OJ}$ that underwent 
OPT and OPV was investigated. In the OPT group, the 7-day survival rate decreased when the occlusion time was $>20 \mathrm{~min}$ (Fig. 1). The safe limit for the duration of liver ischemia for the OPV group was extended to 40 min without affecting the survival rate. At an occlusion time of $50 \mathrm{~min}$, the 7-day survival rate decreased to $90 \%(9 / 10)$ in the OPV group and $0 \%(0 / 10)$ in the OPT group. The 7-day survival rate of the OPV group was significantly increased compared with the OPT group at 40, 50 and 60 min of occlusion, respectively ( $\mathrm{P}<0.05$; Fig. 1). Determined safe limits for the duration of liver ischemia were 20 and $40 \mathrm{~min}$ for the OPT and OPV group, respectively. OPV, with preserved HAF in rats with OJ and partial hepatectomy, markedly prolonged the duration of liver ischemia during hepatectomy.

Hepatic hemodynamic changes in rats with OJ and liver ischemia. Liver PVF and HAF in rats with OJ were measured with an ultrasonic flow meter and compared with those of normal rats. PVF decreased significantly from $13.83 \pm 1.60$ to $8.17 \pm 0.75 \mathrm{ml} / \mathrm{min}$ in rats with $\mathrm{OJ}$ compared to the control $(\mathrm{P}<0.05)$ and HAF increased significantly from $1.10 \pm 0.14$ to $1.98 \pm 0.38 \mathrm{ml} / \mathrm{min}$ in rats with OJ compared with the control ( $\mathrm{P}<0.05$; Fig. 2A). As HAF was low compared with PVF, the total liver blood inflow was decreased 7 days following induction of OJ.

During the portal blood occlusion prior to hepatectomy, $\mathrm{LBF}$ of the right liver lobe was measured with a laser speckle contrast imager. LBF of the control group was $529.53 \pm 91.55$ LSPU (Fig. 2B). Following portal occlusion, LBF significantly decreased to $136.89 \pm 32.32 \mathrm{LSPU}$ for the OPT and $183.99 \pm 49.25 \mathrm{LSPU}$ for the OPV group $(\mathrm{P}<0.05)$. OPT and OPV significantly reduced the perfusion of liver microcirculation, with OPT being more effective compared with OPV $(\mathrm{P}<0.05)$.

Effects of varying hepatic blood inflow occlusions on hepatic biochemical markers and expression of proinflammatory cytokine genes. ALT and DBIL levels of rats with

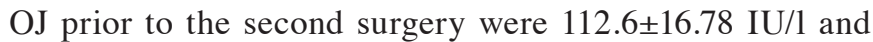
$158.2 \pm 11.38 \mu \mathrm{mol} / 1$, respectively. Following biliary drainage, portal occlusion and hepatectomy, ALT levels in rats with OJ increased, with the value for the OPT group significantly higher compared with the OPV and control groups at 6 and $24 \mathrm{~h}$ of reperfusion $(\mathrm{P}<0.05$; Fig. $3 \mathrm{~A})$. At $24 \mathrm{~h}$ of reperfusion, the ALT level in the OPV group was significantly higher compared with the control group $(\mathrm{P}<0.05)$. Due to the recovery of biliary drainage, DBIL levels in all rats decreased following the second operation, with the value for the OPT group significantly higher compared with the OPV and control groups at $6 \mathrm{~h}$ of reperfusion $(\mathrm{P}<0.05$; Fig. 3B). At days 3 and 7 following hepatectomy, ALT and DBIL levels of all rats had decreased and no significant difference was observed between the groups. Rats in the OPT group suffered the most severe liver I/R injury among the three groups and OPV significantly reduced I/R-induced liver injury compared with the OPT method.

The current study assessed mRNA expression of the proinflammatory cytokines IL-1 $\beta$ and TNF- $\alpha$ (Fig. 3C and D) in the remnant liver tissue using RT-qPCR. mRNA expression of IL-1 $\beta$ and TNF- $\alpha$ was significantly upregulated in the OPT

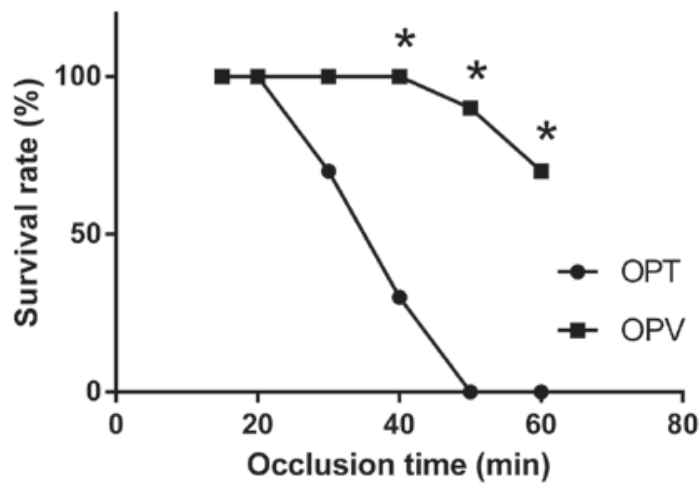

Figure 1. Comparison of the 7-day survival rate of rats with obstructive jaundice that underwent varying types of portal blood occlusion and $76 \%$ hepatectomy. Survival rates were determined following varying occlusion times during the second surgery. $\mathrm{n}=10$ at each time point for each group. ${ }^{*} \mathrm{P}<0.05$ vs. OPT group. OPT, occlusion of the portal triad; OPV, occlusion of the portal vein and preservation of the hepatic artery.

\section{A}

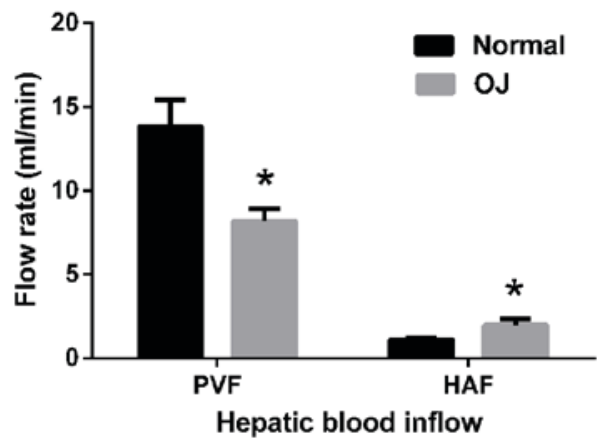

B

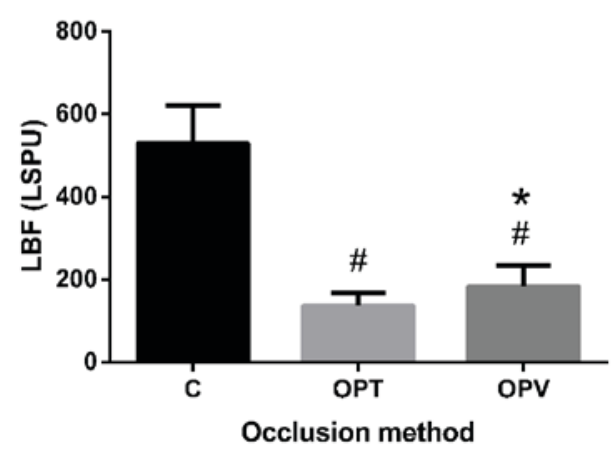

Figure 2. Hepatic hemodynamic changes in rats with OJ. (A) Comparison of liver PVF and HAF in rats with OJ and normal rats by analysis using an ultrasonic flow meter. ${ }^{*} \mathrm{P}<0.05$ vs. the Normal group. (B) Comparison of the microvascular $\mathrm{LBF}$ of rats with OJ during varying methods of portal blood control using a laser speckle contrast imager. ${ }^{~} \mathrm{P}<0.05$ vs. the $\mathrm{C}$ group; ${ }^{*} \mathrm{P}<0.05$ vs. the OPT group. $n=6$ at each time point for each group. PVF, portal vein flow; HAF, hepatic artery flow; OJ, obstructive jaundice; LBF, liver blood flow; C, control without portal blood occlusion; OPT, occlusion of the portal triad; OPV, occlusion of the portal vein and preservation of the hepatic artery.

group compared with the control and OPV groups at 6 and $24 \mathrm{~h}$ following $\mathrm{I} / \mathrm{R}$ and hepatectomy $(\mathrm{P}<0.05)$. The remnant liver in the control and OPV groups expressed similar levels of IL-1 $\beta$ and TNF- $\alpha$ mRNA at all of the time points. The data indicated that OPT induced a more severe inflammatory response and damage compared with OPV. 
A

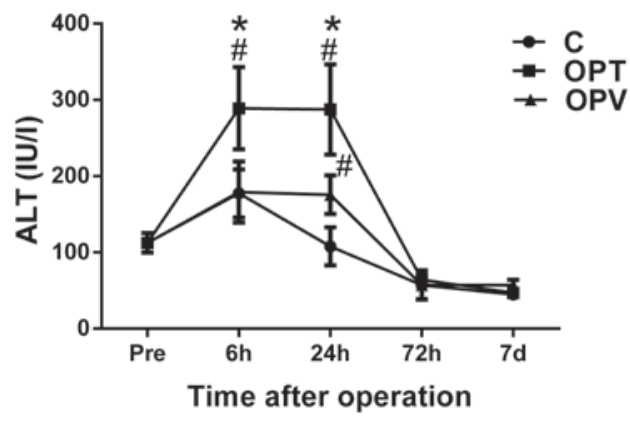

C

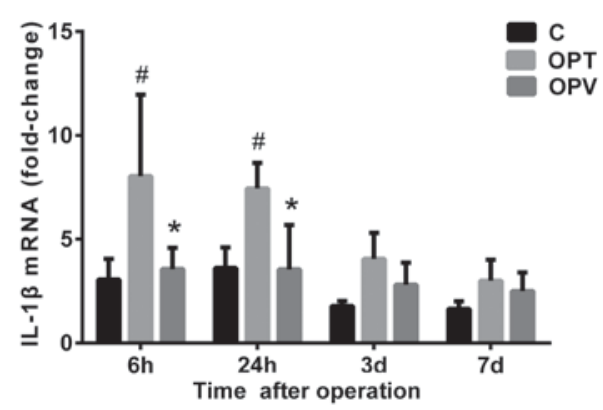

B

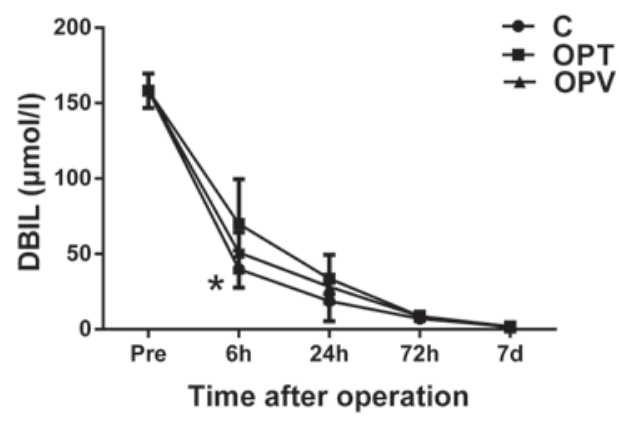

D

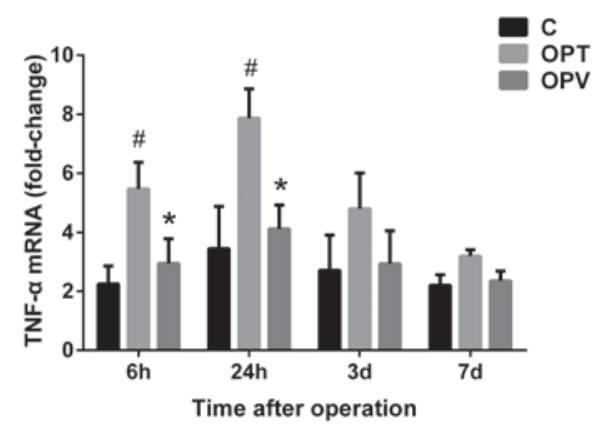

Figure 3. Biochemical markers and expression of proinflammatory cytokine genes. Detection of (A) plasma ALT and (B) DBIL and (C) IL-1 $\beta$ and (D) TNF- $\alpha$ mRNA expression over time in rats with obstructive jaundice that underwent varying intraoperative hepatic blood inflow occlusions and hepatectomy. $\mathrm{n}=6$ at each time point for each group. ${ }^{\#} \mathrm{P}<0.05$ vs. the $\mathrm{C}$ group; ${ }^{*} \mathrm{P}<0.05$ vs. the OPT group. $\mathrm{C}$, control without portal blood occlusion; OPT, occlusion of the portal triad; OPV, occlusion of the portal vein and preservation of the hepatic artery; ALT, alanine transaminase; DBIL, direct bilirubin; IL-1 $\beta$, interleukin-1 $\beta$; TNF- $\alpha$, tumor necrosis factor- $\alpha$.

Pathological changes in the liver of rats with OJ following varying methods of portal occlusion and partial hepatectomy. Hepatic pathological changes in rats with $\mathrm{OJ}$ in the $\mathrm{C}$, OPT and OPV groups were assessed at 6, 24 and $72 \mathrm{~h}$ and 7 days following different portal occlusion procedures and hepatectomy (Fig. 4). Proliferative encysted cholangioles were observed in all groups, with prevalence at 6 and $24 \mathrm{~h}$ following the second surgical procedure. In addition, marked hepatocyte necrosis was detected in the OPT group with many neutrophils invading into the necrotic area at 6 and $24 \mathrm{~h}$ post hepatectomy. The necrotic area was enlarged at $72 \mathrm{~h}$ following the second surgical procedure. No necrotic area was observed in the $\mathrm{C}$ group and hepatocyte necrosis in the OPV group was minimal when compared with the OPT group. OPV was effective in preventing hepatocytic necrosis in rats with OJ, I/R and partial hepatectomy.

Liver regeneration in rats with $O J$ that underwent partial hepatectomy and varying portal occlusion procedures. Regeneration of the remnant liver following different intraoperative hepatic blood inflow occlusion procedures and partial hepatectomy were evaluated on days 3 and 7 following I/R and hepatectomy. Rates of liver regeneration on day 3 post-hepatectomy of C, OPV and OPT groups were $69.27 \pm 4.97$, $66.18 \pm 4.43$ and $59.70 \pm 5.03 \%$, respectively (Fig. 5A). The rate of hepatic regeneration of the OPT group was significantly lower compared with the $\mathrm{C}$ and OPV groups $(\mathrm{P}<0.05)$, indicating that total hepatic pedicle clamping for $30 \mathrm{~min}$ prior to hepatectomy

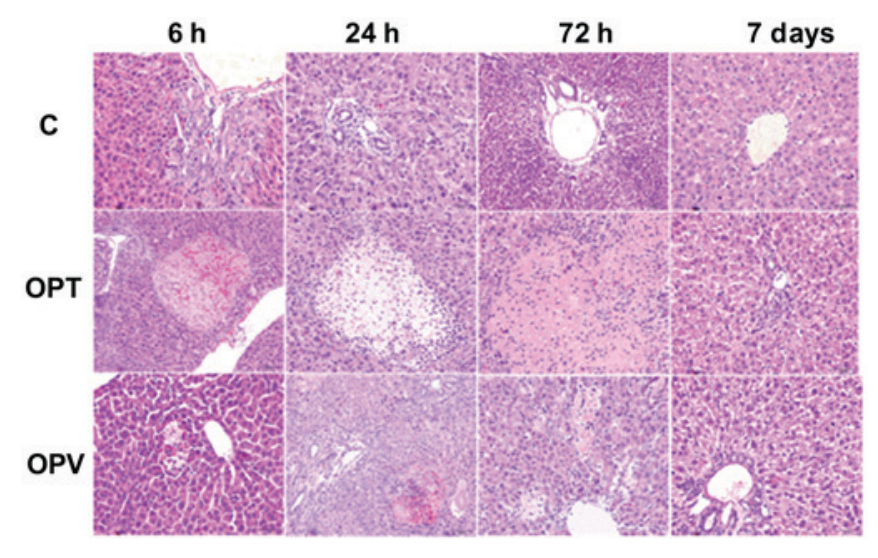

Figure 4. Representative hematoxylin and eosin staining of the remnant liver of rats with obstructive jaundice that underwent varying intraoperative hepatic blood inflow occlusions at 6,24 and $72 \mathrm{~h}$ and 7 days following liver ischemia-reperfusion and partial hepatectomy. Visualized using light microscopy (magnification, $\mathrm{x} 400$ ). C, control group without portal blood occlusion; OPT, occlusion of the portal triad; OPV, occlusion of the portal vein and preservation of the hepatic artery.

inhibited liver regeneration. Preservation of HAF during hepatic blood inflow occlusion in the OPV group did not significantly reduce liver regeneration compared with the control group. On day 7 following the second surgical procedure, rates of liver regeneration of C, OPV and OPT reached 96.17 \pm 3.84 , $94.23 \pm 5.44$ and $91.33 \pm 9.29 \%$, respectively. No statistical differences were detected among the groups. 
A

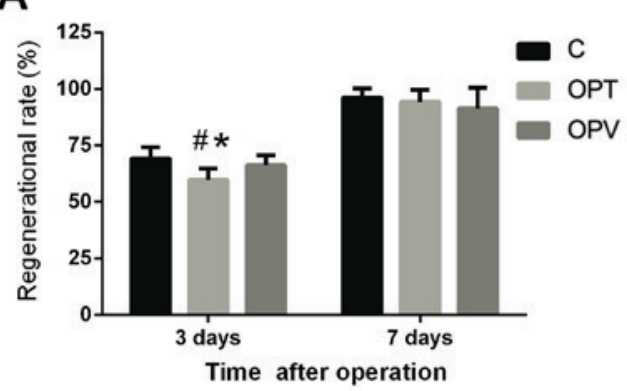

C
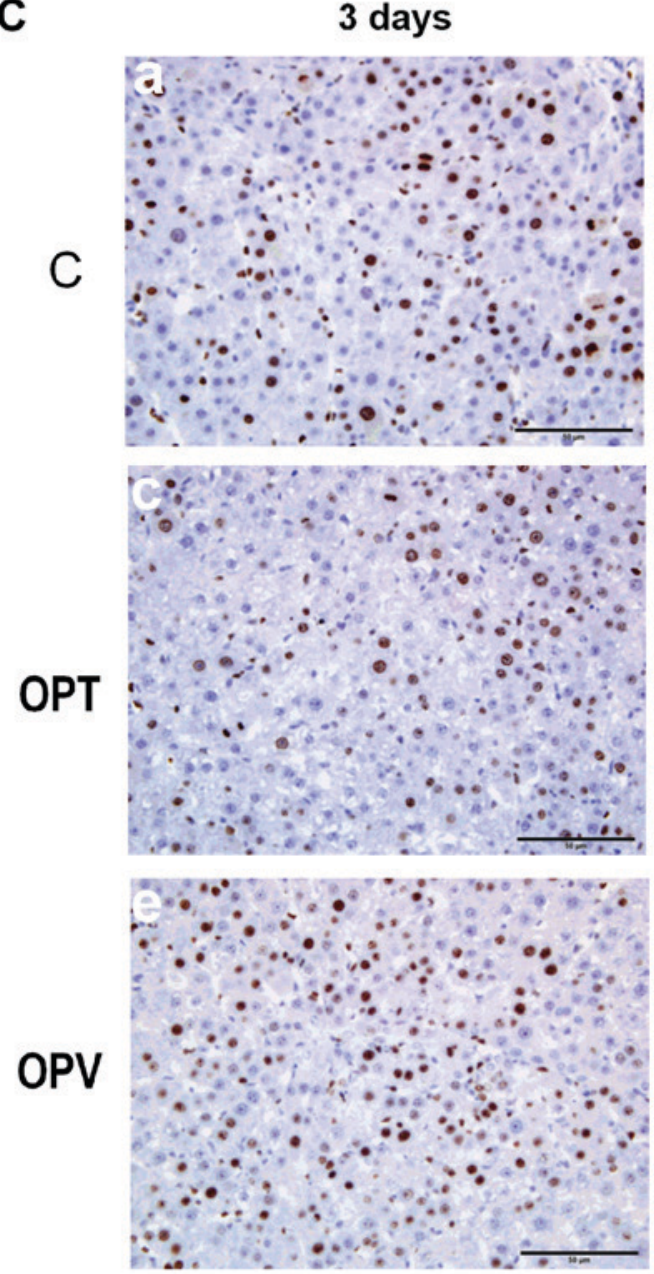

B

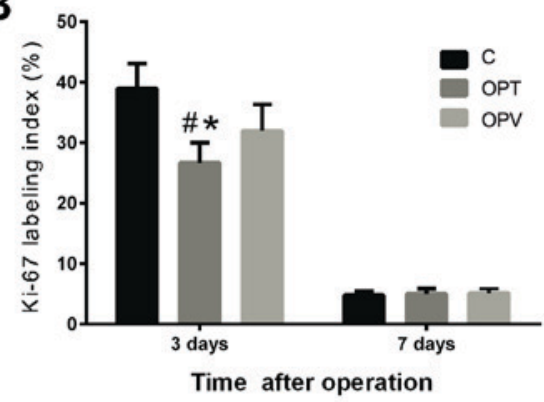

7 days
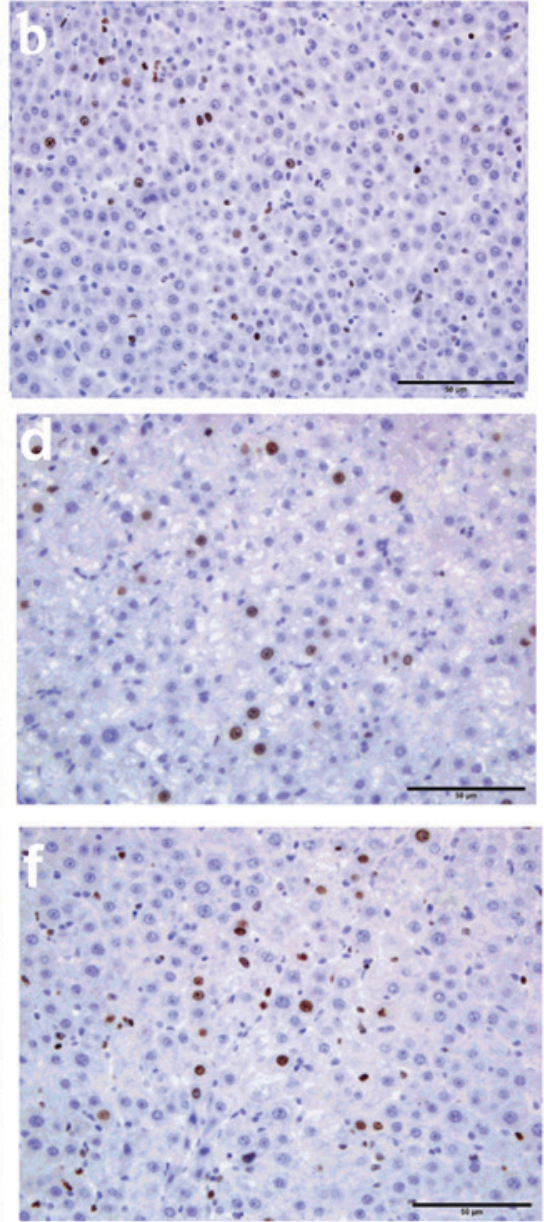

Figure 5. Liver regeneration and Ki-67 expression in rats with obstructive jaundice that underwent varying intraoperative hepatic blood inflow occlusions on days 3 and 7 following ischemia-reperfusion and hepatectomy. (A) Rate of liver regeneration calculated using liver weight. (B) Ki-67 labeling index. (C) Representative light microscopy image of immunohistochemical staining of Ki-67 in liver sections (magnification, $\mathrm{x} 400$ ). ${ }^{*} \mathrm{P}<0.05$ vs. the $\mathrm{C}$ group; ${ }^{*} \mathrm{P}<0.05$ vs. the OPV group. $\mathrm{n}=6$ at each time point for each group. $\mathrm{C}$, control group without portal blood occlusion; OPT, occlusion of the portal triad; OPV, occlusion of the portal vein and preservation of the hepatic artery.

In order to further characterize the liver regenerative response, expression of $\mathrm{Ki}-67$ is indicated as a brown nucleus in immunostained tissue sections, a nuclear antigen that is associated with hepatocyte proliferation, was assessed in remnant liver tissues. On day 3 post-operation, Ki-67 labeling indices of the OPT group $(26.68 \pm 3.27 \%)$ were significantly reduced compared with the C group (38.96 $\pm 4.14 \%)$ and the OPV group (31.94 $\pm 4.38 \%)$ $(\mathrm{P}<0.05$; Fig. 5A) with the OPV group also lower than the $\mathrm{C}$ group $(\mathrm{P}<0.05$; Fig. 5B and $\mathrm{C}$ ). On day 7 following hepatectomy, $\mathrm{Ki}-67$ expression was substantially decreased and no significant differences were detected among the groups (Fig. 5B and C).
Effects of varying liver blood inflow occlusion procedures on hepatocyte proliferation and apoptosis. To further assess the liver regenerative capacity of rats with OJ following hepatectomy with varying liver blood inflow occlusive methods, PCNA, caspase-3 and 9 expression was determined by western blotting (Fig. 6). PCNA is a marker that is associated with hepatocyte proliferation and caspase- 3 and -9 are associated with hepatocyte apoptosis $(21,22)$.

PCNA expression increased at $24 \mathrm{~h}$ following I/R and hepatectomy in all groups compared with the $6 \mathrm{~h}$ measurements. PCNA expression at $24 \mathrm{~h}$ in group $\mathrm{C}$ was markedly 
A

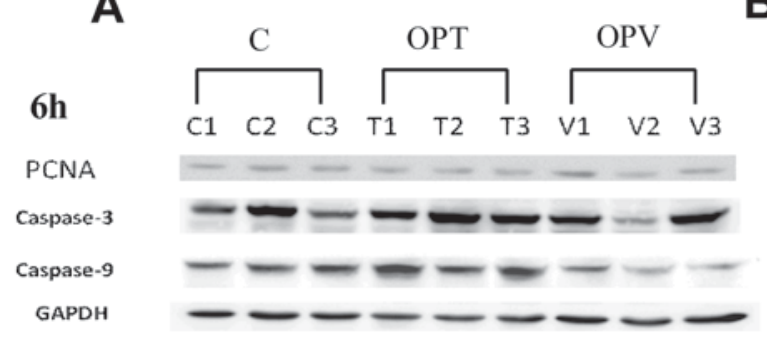

C
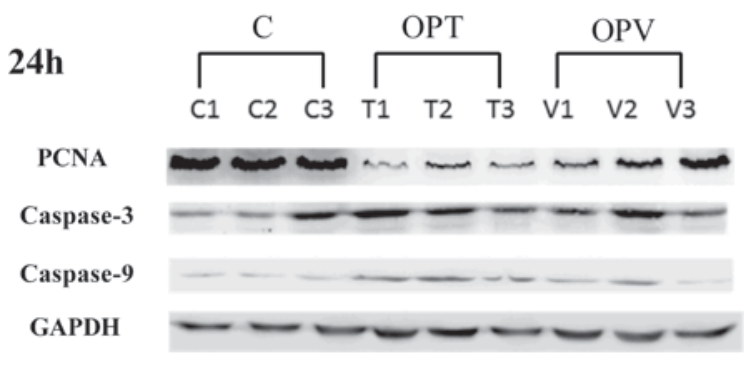

E

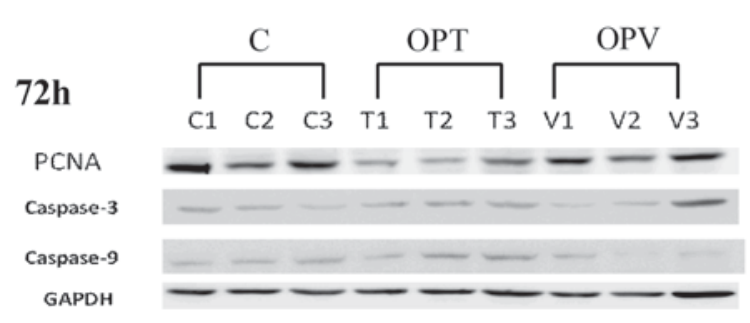

B

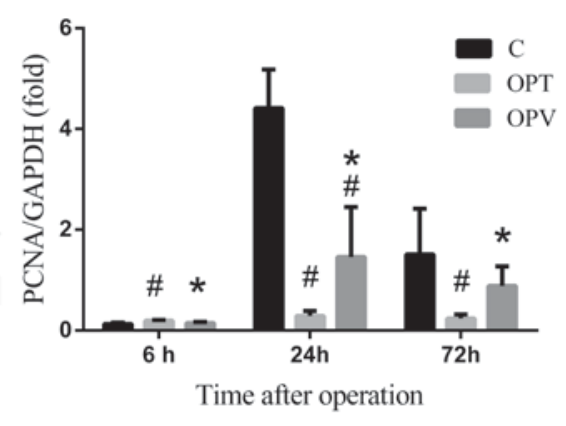

D

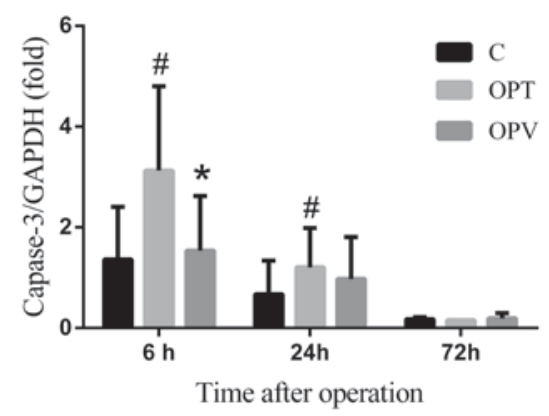

$\mathbf{F}$

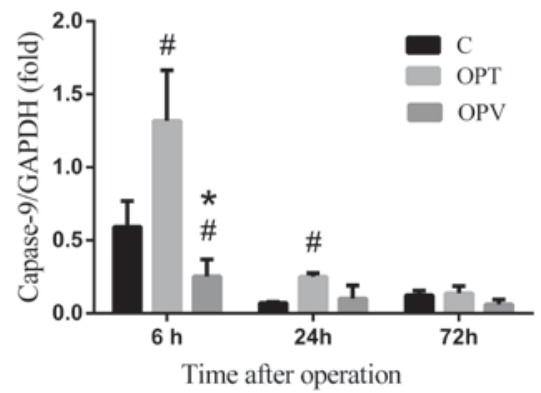

Figure 6. Protein expression in the remnant liver of rats with obstructive jaundice that underwent different intraoperative hepatic blood inflow occlusions and hepatectomy. Western blot analysis of PCNA, caspase-3 and 9 expressions at (A) $6 \mathrm{~h}$, (C) $24 \mathrm{~h}$ and (E) $72 \mathrm{~h}$ following hepatectomy. The quantified results of (B) PCNA, (D) caspase-3 and (F) caspase-9 are presented. ${ }^{*} \mathrm{P}<0.05$ vs. the $\mathrm{C}$ group; ${ }^{*} \mathrm{P}<0.05$ vs. the OPT group. $\mathrm{n}=3$ rats per group per time point. C, control group without portal blood occlusion; OPT, occlusion of the portal triad; OPV, occlusion of the portal vein and preservation of the hepatic artery; PCNA, proliferating cell nuclear antigen.

higher compared with OPT and OPV groups. PCNA expression in the OPV group was significantly increased compared with the OPT group at 24 and $72 \mathrm{~h}$ following hepatectomy $(\mathrm{P}<0.05$; Fig. 6). The opposite pattern was detected for caspase- 3 and 9 expression, where expression in the OPT group was higher compared with the OPV group at 6 and $24 \mathrm{~h}$ following I/R and hepatectomy ( $\mathrm{P}<0.05$; Fig. 6$)$. The results demonstrated that OPV was effective in improving hepatocyte proliferation and reducing hepatocyte apoptosis in rats with OJ following hepatectomy.

\section{Discussion}

The Pringle maneuver is frequently used in hepatectomy due to its effectiveness in reducing blood loss during operation $(4,6,7)$. However, I/R injury is inevitable in patients that require massive hepatectomy or in those with poor liver function, including cirrhosis and jaundice $(5,7)$. Several strategies, including intermittent Pringle maneuver, selective hemi-hepatic vascular occlusion and ischemic preconditioning, have been proposed to improve the tolerance of the remnant liver to I/R injury (11-13,23). However, none of these methods has been accepted as an ideal technique that results in reductions in liver injury and blood loss. It has previously been reported that OPV and preservation of HAF can significantly reduce hepatic I/R injury without increasing the risk of blood loss (14). This new surgical procedure provides better liver cytoprotection compared with OPT. In a previous study with $\mathrm{CCl}_{4}$-induced cirrhotic rats, OPV alone was also demonstrated to be effective in decreasing liver injury (24). 
$\mathrm{OJ}$ is frequently reported in hepatobiliary surgery. The risk of liver failure is greater in individuals with jaundice compared with those without, due to pathological changes in jaundice, including cholangitis and cholestatic liver injury $(25,26)$. Therefore, the current study investigated effects of the OPV method for controlling liver blood inflow and reducing liver injury in rats with OJ and hepatectomy.

As is demonstrated in the present study, total LBF of rats with OJ was markedly decreased compared with the normal rats. Although HAF of the OJ rats was increased, total LBF of the hepatic artery and portal vein decreased due to a decrease in PVF. This hemodynamic change in the liver of rats with OJ may result from compressed and damaged terminal parts of the portal vein and the hepatic sinusoid, which was due to the swelling of the OJ (26). This may account for the observation that rats with OJ have a decreased tolerance for hepatic portal occlusion. Following portal occlusion, LBF decreased significantly in both OPT and OPV groups. In a previous study it was demonstrated that the preservation of the hepatic artery when performing OPV may reduce I/R injury by supplying more oxygen to the liver and without markedly increasing blood loss compared with the Pringle maneuver in normal rats (14).

To the best of our knowledge, the present study is the first to determine the safe limit for the duration of liver ischemia as $20 \mathrm{~min}$ for the OPT and $40 \mathrm{~min}$ for the OPV method in rats with $\mathrm{OJ}$. In addition, prolonging the occlusion time to 50 min significantly decreased the 7-day survival rate of the OPV group compared with the OPT group. Following portal occlusion and hepatectomy, ALT levels in the OPT group significantly increased and peaked at 6 and $24 \mathrm{~h}$ post-operation. ALT levels in the OPV and control groups were similar, which was consistent with the hepatic histological changes that were observed.

In order to further investigate the molecular changes in the liver following hepatectomy with OJ and portal blood occlusion, TNF- $\alpha$ and IL-1 $\beta$ expression in the remnant liver were analyzed. It was revealed that TNF- $\alpha$ and IL-1 $\beta$ mRNA expression in the OPT group was significantly higher compared with the control and OPV groups. The results demonstrated that preserving HAF may significantly reduce the inflammatory response compared with the OPT method, which is generally produced by I/R-activated inflammatory cells (27). In a previous study, it was demonstrated that liver tissue malondialdehyde values were significantly lower and $\mathrm{Na}^{+}-\mathrm{K}^{+}$-ATPase activity was significantly higher in the OPV group compared with the OPT group (14). An imbalance between the expression of genes that are involved in vasoconstriction and vasodilation was also observed in the OPT group, but not in the OPV group (28). Preserving low liver blood perfusion using the OPV method during liver surgery may be very effective for preventing hepatic microcirculatory dysfunction and hepatocyte injury.

When evaluating the advantages and disadvantages of a method for portal occlusion, the effect on hepatic regeneration is a vital factor. Hepatobiliary surgeons may select a method for occlusion that improves the liver regenerative ability. Following portal blood occlusion and hepatectomy of the same volumes of tissues, hepatic regeneration of the OPV group was significantly improved compared with the OPT group. At day 3 post-occlusion and hepatectomy, rates of liver regeneration and Ki-67 labeling indices of the OPV group were significantly higher compared with the OPT group. The PCNA level in the OPV group was higher compared with the OPT group according to western blot results. As indicated by the results presented in the current study, OPV improved the time of liver regeneration in rats with OJ compared with the OPT group. Combined with findings from laser speckle contrast imaging, low perfusion of oxygen in the hepatic artery blood and reduced damage in the OPV group may be key factors for the observed improvements in liver regeneration.

Overall, preserving HAF during portal triad blood occlusion may significantly reduce I/R injury and improve liver regeneration in rats with $\mathrm{OJ}$ that underwent partial hepatectomy compared with the Pringle maneuver, which was performed in the OPT group. The findings may provide guidance for future clinical studies. It may decrease the risk of liver failure following major hepatectomy, which is on occasion inevitable and potentially fatal when the duration of hepatic inflow occlusion is prolonged and resection of large volumes of liver tissue is necessary in order to clear niduses, particularly in patients with jaundice that present with hilar cholangiocarcinoma (29). A limitation of the OPV method in clinical application is the need to isolate the portal vein for occlusion by dissociating the pedicle of Glisson's capsule. In addition, auxiliary measures, including intraoperative low central venous pressure and appliance of ligature, may be used at the same time to reduce the blood loss in the application of OPV method in OJ patients who need a hepatectomy.

In conclusion, to the best of our knowledge, this is the first study on preserving HAF during portal triad blood clamping in the liver of rats with OJ. The OPV method resulted in less damage to the liver and it may facilitate improved liver regeneration. It may increase the current limits on the duration of portal blood occlusion. The OPV method may describe a novel technique of choice for controlling hepatic blood inflow in liver surgery, particularly in patients with complex jaundice cases, due to its simplicity and as it may be safer compared with the Pringle maneuver.

\section{Acknowledgements}

Not applicable.

\section{Funding}

This work was supported by the Project of the National Natural Science Foundation of China (grant no. 81271738) and the National Key Technology R\&D Program of China (grant no. 2012BAI06B01).

\section{Availability of data and materials}

All data generated or analyzed during this study are included in this published article.

\section{Authors' contribution}

ZK contributed to the animal experiments, data collection and analysis. $\mathrm{JJH}$ contributed to the interpretation of the data, writing and editing the article. XLG and KP contributed to data analysis and histopathology examinations. 
JHD contributed to conception and design. CHL contributed to conception, writing, critical revision and editing of the article.

\section{Ethics approval and consent to participate}

The current study was approved by the Ethical and Research Committee of the Chinese PLA Medical School (Beijing, China).

\section{Patient consent for publication}

Not applicable.

\section{Competing interests}

The authors declare that they have no competing interests.

\section{References}

1. Brandi G, Venturi M, Pantaleo MA and Ercolani G; GICO: Cholangiocarcinoma: Current opinion on clinical practice diagnostic and therapeutic algorithms: A review of the literature and a long-standing experience of a referral center. Dig Liver Dis 48: 231-241, 2016.

2. Uppal DS and Wang AY: Advances in endoscopic retrograde cholangiopancreatography for the treatment of cholangiocarcinoma. World J Gastrointest Endosc 7: 675-687, 2015.

3. Kim YI: Ischemia-reperfusion injury of the human liver during hepatic resection. J Hepatobiliary Pancreat Surg 10: 195-199, 2003.

4. van Riel WG, van Golen RF, Reiniers MJ, Heger M and van Gulik TM: How much ischemia can the liver tolerate during resection? Hepatobiliary Surg Nutr 5: 58-71, 2016.

5. Celotti A, Solaini L, Montori G, Coccolini F, Tognali D and Baiocchi G: Preoperative biliary drainage in hilar cholangiocarcinoma: Systematic review and meta-analysis. Eur J Surg Oncol 43: 1628-1635, 2017.

6. Man K, Fan ST, Ng IO, Lo CM, Liu CL and Wong J: Prospective evaluation of Pringle maneuver in hepatectomy for liver tumors by a randomized study. Ann Surg 226: 704-711, 1997.

7. van Gulik TM, de Graaf W, Dinant S, Busch OR and Gouma DJ: Vascular occlusion techniques during liver resection. Dig Surg 24: 274-281, 2007.

8. Hammond JS, Guha IN, Beckingham IJ and Lobo DN: Prediction, prevention and management of postresection liver failure. Br J Surg 98: 1188-1200, 2011.

9. Hameed A, Pang T, Chiou J, Pleass H, Lam V, Hollands M, Johnston E, Richardson A and Yuen L: Percutaneous vs. endoscopic pre-operative biliary drainage in hilar cholangiocarcinoma-a systematic review and meta-analysis. HPB (Oxford) 18: 400-410, 2016.

10. Blechacz B, Komuta M, Roskams T and Gores GJ: Clinical diagnosis and staging of cholangiocarcinoma. Nat Rev Gastroenterol Hepatol 8: 512-522, 2011.

11. Li M, Zhang C, Zhang T, Wang L, Ding Y, Niu Z, He S and Yang Z: Outcome using selective hemihepatic vascular occlusion and Pringle maneuver for hepatic resection of liver cavernous hemangioma. World J Surg Oncol 13: 267, 2015.

12. Dou L, Meng WS, Su BD, Zhu P, Zhang W, Liang HF, Chen YF and Chen XP: Step-by-step vascular control for extracapsular resection of complex giant liver hemangioma involving the inferior vena cava. Am Surg 80: 15-20, 2014.

13. Gurusamy KS, Kumar Y, Pamecha V, Sharma D and Davidson BR: Ischaemic pre-conditioning for elective liver resections performed under vascular occlusion. Cochrane Database Syst Rev: CD007629, 2009.
14. Chen YW, Li CH, Zhang AQ, Yang SZ, Zhang WZ and Dong JH: Preserving hepatic artery flow during portal triad blood inflow occlusion reduces liver ischemia-reperfusion injury in rats. J Surg Res 174: 150-156, 2012.

15. Sakaguchi K, Takeuchi E, Suzuki M, Oda K, Nagino M, Nimura Y and Yoshida S: DNA polymerases and Ki-67 nuclear antigen are induced in correlation with the resected mass of rat liver up to 90\%. Langenbecks Arch Surg 385: 135-142, 2000.

16. Institute for Laboratory Animal Research: Guide for the care and use of laboratory animals, 8th (ed.). Washington (DC): National Academies Press (US), 2011.

17. Huang X, Li CH, Zhang AQ, Kong Z, Gu WQ and Dong JH: A simple rat model of in situ reversible obstructive jaundice in situ reversible obstructive jaundice model. Ann Surg Treat Res 92: 389-395, 2017.

18. Li CH, Wang HD, Hu JJ, Ge XL, Pan K, Zhang AQ and Dong JH: The monitoring of microvascular liver blood flow changes during ischemia and reperfusion using laser speckle contrast imaging. Microvasc Res 94: 28-35, 2014.

19. Livak KJ and Schmittgen TD: Analysis of relative gene expression data using real-time quantitative PCR and the 2(-Delta Delta C(T)) method. Methods 25: 402-408, 2001.

20. Wang PF, Li CH, Chen YW, Zhang AQ, Cai SW and Dong JH: Preserving hepatic artery flow during portal triad blood inflow occlusion improves remnant liver regeneration in rats after partial hepatectomy. J Surg Res 181: 329-336, 2013.

21. Paranjpe S, Bowen WC, Bell AW, Nejak-Bowen K, Luo JH and Michalopoulos GK: Cell cycle effects resulting from inhibition of hepatocyte growth factor and its receptor c-Met in regenerating rat livers by RNA interference. Hepatology 45: 1471-1477, 2007.

22. Rehman H, Sun J, Shi Y, Ramshesh VK, Liu Q, Currin RT, Lemasters JJ and Zhong Z: NIM811 prevents mitochondrial dysfunction, attenuates liver injury, and stimulates liver regeneration after massive hepatectomy. Transplantation 91: 406-412, 2011.

23. Fu SY, Lau WY, Li GG, Tang QH, Li AJ, Pan ZY, Huang G, Yin L, Wu MC, Lai EC and Zhou WP: A prospective randomized controlled trial to compare Pringle maneuver, hemihepatic vascular inflow occlusion, and main portal vein inflow occlusion in partial hepatectomy. Am J Surg 201: 62-69, 2011.

24. Hu JJ, Li CH, Wang HD, Xu WL, Zhang AQ and Dong JH: Portal vein clamping alone confers protection against hepatic ischemia-reperfusion injury via preserving hepatocyte function in cirrhotic rats. J Surg Res 194: 139-146, 2015.

25. Nakanishi Y, Tsuchikawa T, Okamura K, Nakamura T, Tamoto E, Noji T, Asano T, Amano T, Shichinohe T and Hirano S: Risk factors for a high Comprehensive Complication Index score after major hepatectomy for biliary cancer: A study of 229 patients at a single institution. HPB (Oxford) 18: 735-741, 2016.

26. Xin KY, Yee LS, Yong TT and Fui AC: Obstructive jaundice due to intraductal tumour thrombus in recurrent hepatocellular carcinoma: What is the optimal therapeutic approach? Hepatogastroenterology 61: 1863-1866, 2014.

27. Wanner GA, Ertel W, Müller P, Höfer Y, Leiderer R, Menger MD and Messmer K: Liver ischemia and reperfusion induces a systemic inflammatory response through Kupffer cell activation. Shock 5: 34-40, 1996.

28. Li CH, Chen YW, Chen YL, Yao LB, Ge XL, Pan K, Zhang AQ and Dong JH: Preserving low perfusion during surgical liver blood inflow control prevents hepatic microcirculatory dysfunction and irreversible hepatocyte injury in rats. Sci Rep 5: 14406, 2015.

29. Wiggers JK, Groot Koerkamp B, Cieslak KP, Doussot A, van Klaveren D, Allen PJ, Besselink MG, Busch OR, D'Angelica MI, DeMatteo RP, et al: Postoperative mortality after liver resection for perihilar cholangiocarcinoma: Development of a risk score and importance of biliary drainage of the future liver remnant. J Am Coll Surg 223: 321-331.e1, 2016.

(i) (5) This work is licensed under a Creative Commons Attribution-NonCommercial-NoDerivatives 4.0 International (CC BY-NC-ND 4.0) License. 